\title{
Metrological characterization of new infrared sensors for robot navigation
}

\author{
Mazierli D., Zanobini A., University of Florence, Information Engineering Department, Via di Santa Marta 3, 50139 Florence, Italy
}

waves [15],

\begin{abstract}
UAV role in our society is increasingly important and the number of flying drones is exponentially growing. Safety related problems must be considered and the autonomous navigation is a grand challenge for engineers. This article reports on first experiences using state-of-the-art, time-offlight, infrared sensors in outdoor environment. Speed and lightness make these sensors suitable for ground and especially for aerial robot navigation. Range, accuracy and precision of different modes and different sensors will be compared.
\end{abstract}

Keywords - Infrared sensor, Anti-collision system, Drone, Measurements.

\section{INTRODUCTION}

Unmanned Aerial Vehicles (often referred as drones) were born for war purposes, but in the last years they are spreading in many civilian applications. Thanks to the high versatility and relatively low cost, drones can be used in wide-ranging activities from internet delivery [1] [2] to rescue operations. Drones are often equipped with sensors in order to map and study the area in their field of view, then it is possible to use them for photogrammetry and land mapping [3], gas leakages monitoring [4], farming [5] or environmental monitoring such as forest fire monitoring [6]. As they are able to fly, drones are suitable for security [7], reconnaissance [8], surveillance [9] [10], or they can be equipped with cameras for professional and entertainment scopes. An interesting paper [11] shows how drones could be used to create a layered network, called Internet of Drones, similar to the existing modern networks. Anyway, the number of flying drones is expected to grow and also the risk of collisions will increase with traffic.

For all the mentioned applications, a reliable anticollision system is suitable in order to avoid collisions with objects or other drones during operations [12]. Since many years engineers are developing advanced automatic systems to allow drones avoiding different obstacles [13] [14]. It is clear that an anti-collision system is based on distance measurements and this is

the reason why a distance sensor is the primary element to avoid collision. The sensor can use radio infrared [16] [17], light [18] or ultrasounds [19], but it must be light and fast. The lightness is requested in order to increase battery life and flight autonomy; high update frequency of data is necessary for rapid changing distances. A recent study shows an efficient algorithm for collision avoidance based on RGB camera [20].

\section{SENSORS UNDER TEST}

The TeraRangerOne is an infrared sensor made by Terabee, a start-up placed in Saint-Genis-Pouilly, France. This sensor uses three infrared LEDs to emit pulses of light and a central detector to receive the reflected waves. The elapsing time between emission and detection is the time-of-flight (TOF) and it is used to get indirect measurements of distance. The sensor it's only $8 \mathrm{~g}$ and its update frequency can go up to 1 $\mathrm{kHz}$. Combined with high range and resolution, good accuracy and low power consumption, these features make the sensor a perfect choice for drones' anticollision system [21]. TeraRangerOne can works in three different modes: the precision mode, which is suitable when one needs good accuracy, the fast mode, which is suitable when one needs high update rate, and the outdoor mode, recently loaded and designed for outdoor environments. CERN published a paper [22] with the results of some tests on TeraRangerOne and other sensors of similar scope, but only using the fast and the precision mode, because the outdoor mode didn't exist. Recently, not only the company uploaded the new mode, but also developed an outdoor version of the TeraRangerOne, which has the same three modes of the "classic" version (from now as defined as "indoor version"), the same technology, but a revolutionary hardware that allows the sensor to fit any condition of an outdoor environment. Currently, there are only some prototypes of the new sensor and so far, a specific calibration firmware was not developed: the calibration process [16] of the outdoor version is the same of the indoor one. The goals of the tests discussed in this paper are basically two:

- To understand if it's really necessary to develop an outdoor version of the TeraRangerOne or if the recent introduction of an outdoor mode applied to the indoor version can be sufficient to get distance measurements 
in any outdoor environment.

- In case a specific sensor for the outdoor should be needed, to understand if the calibration firmware used for the indoor version could be used also for the outdoor one or if a new specific firmware is required.

In order to answer these questions many tests were performed in different environmental conditions, with different targets, using both sensors in all their three modes.

\section{CHOICE OF THE TARGETS}

The first step was the choice of the targets from which to measure the distance. The selection was not casual, but it has been performed by considering the applications of the sensor, specifically which materials it could meet when it will be sold and used. Furthermore, targets with different optical characteristics were selected.

Using an IR-sensitive camera it is possible to see how much an object absorbs or reflects the infrared light, and therefore also how difficult or easy is to detect it with an infrared sensor who needs a reflected signal [23]. Considering this, four targets were chosen: an irregular wood wall (Figure 1), a white gate (Figure 2), a dark polished car (Figure 3) and a glass door (Figure 4).
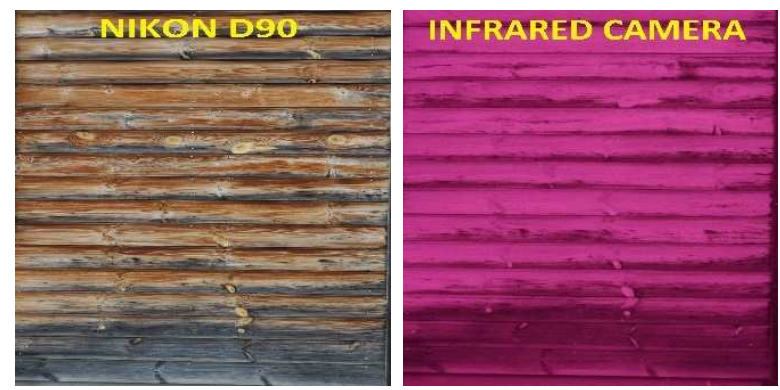

Figure 1: picture taken by a normal camera (left) compared with one taken by an infrared camera (right)

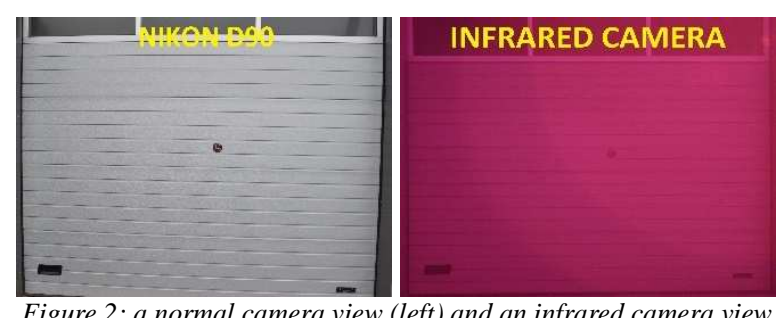

Figure 2: a normal camera view (left) and an infrared camera view (right) of a white gate
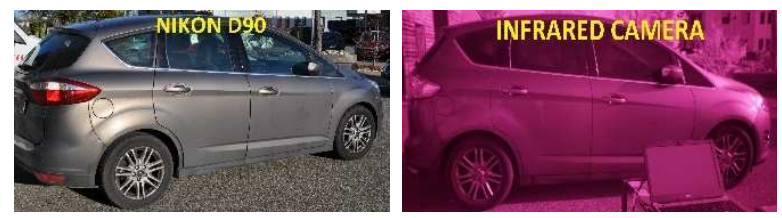

Figure 3: how a dark grey car appears in the two different areas of the spectrum

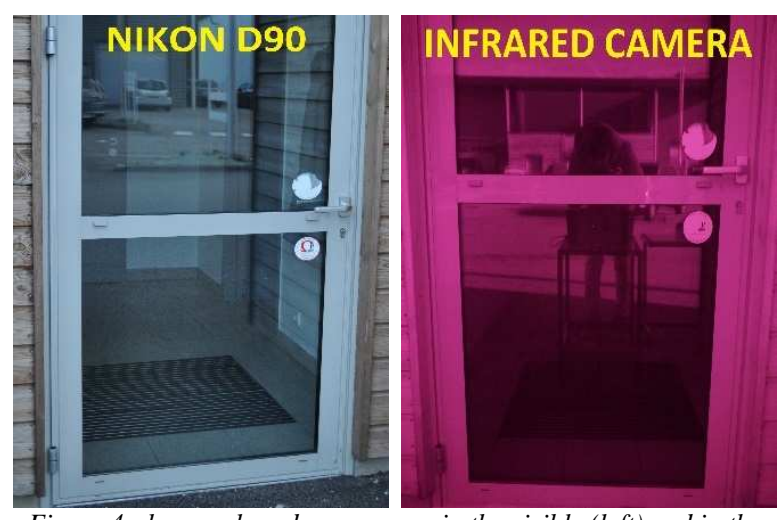

Figure 4: how a glass door appears in the visible (left) and in the infrared (right)

These pictures deserve a careful look as they contain some important information. For example, comparing Figure 1 and Figure 2, one can note that the two targets are very different in the visible spectrum, but their infrared characteristics are pretty similar. It is not always possible to deduce the infrared reflectivity of an object only by eye observation: it can happen that a black thing (that absorbs all the wavelengths perceptible by the human sight) is very reflective in the infrared area and may appear as white to an infrared camera. In one simple phrase: the infrared characteristics of an object are not closely related to its colour.

For the TeraRangerOne, not all the targets are equally easy to be detected, as this depends on the infrared reflection of the targets: its range is inversely proportional to the infrared absorption of the target.

\section{DATA ACQUISITION}

After choosing the targets, data have been acquired. The protocol included data acquisition at the following distances: $1 \mathrm{~m}, 3 \mathrm{~m}, 4 \mathrm{~m}, 5 \mathrm{~m}, 7 \mathrm{~m}, 9 \mathrm{~m}, 11 \mathrm{~m}, 13 \mathrm{~m}, 14 \mathrm{~m}$; in this order and with possible additions or variations depending on the type of the target.

Figure 5 shows the equipment used to perform the measurements.

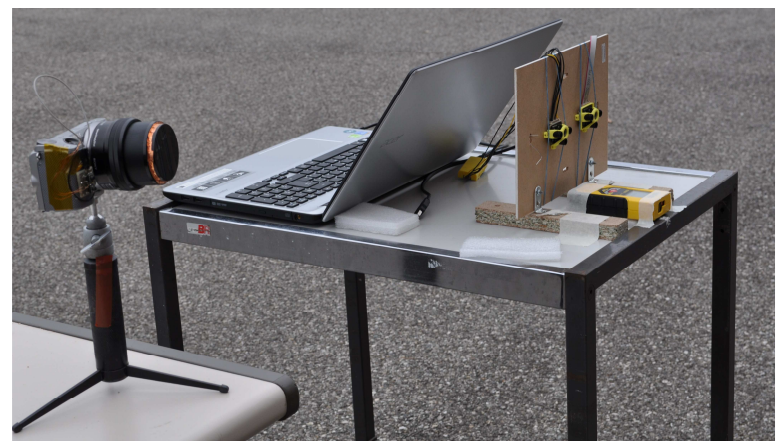

Figure 5: infrared camera, PC, sensors and laser measuring: positions for the test

The sensors have been placed on a rigid surface fixed 
on a mobile cart a few centimeters away from each other, so that the light emitted by the sensors could be perpendicular to the target. A laser measure was used to check the distance from which data were acquired. Through the application hterm it was possible to read data coming from the sensor, while Minitab was utilized for statistical analysis.

It is important to make a clarification before showing the results of the test: nowadays beyond the $14 \mathrm{~m}$ no calibration exists for the Terabee sensors, because until a few months ago beyond this threshold the sensors didn't receive the back signal. Everything has changed with the introduction of the outdoor mode and of the outdoor version of TeraRangerOne, and now both sensors are able to go beyond $20 \mathrm{~m}$ in some conditions but, as already mentioned, the sensors are not calibrated for distances greater than $14 \mathrm{~m}$, therefore all data readings are meaningless and we decided not to show them.

\section{INDOOR SENSOR - OUTDOOR MODE}

The outdoor mode has only recently been added to the sensors and thus this mode has never been tested. The first goal is to understand if the outdoor mode has increased the quality of the sensor compared to when it had only precision and fast modes.

It is possible to answer this question by analysing the graphs that compare the measurements of one target in the same environmental conditions, in the different modes.

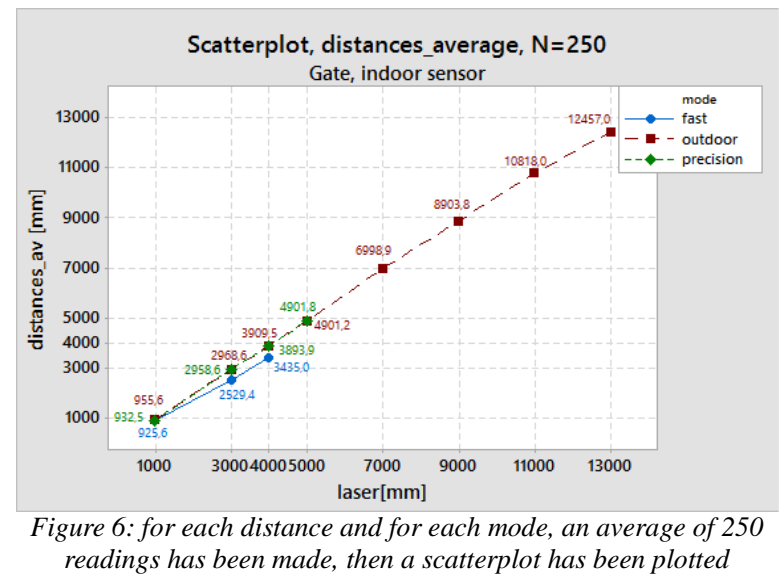

Figure 6 shows the average values of 250 readings of the indoor sensor for each distance and for each mode. This scatterplot clearly highlights the differences in range between the three modes: the fast mode has only a 4 meters range, the precision mode goes up to 5 meters, the outdoor mode goes beyond 13 meters. Another characteristic that can be qualitatively noted in Figure 6 is the linearity of the sensor, that fails in fast mode. Figure 7 has been utilized to compare the accuracy of the three modes.

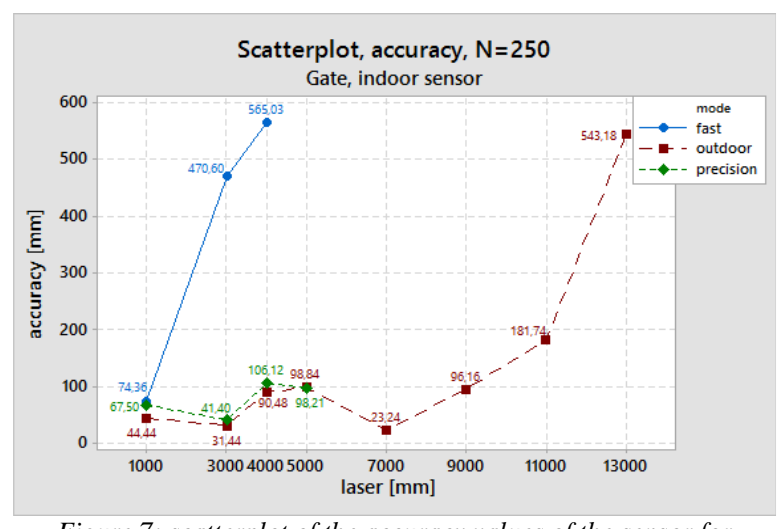

Figure 7: scatterplot of the accuracy values of the sensor for various distances and for the three modes

Accuracy is defined as the maximum deviation of the measured displacement with respect to the reference value. The measured value is obtained by an average of 250 sequential values reading.

Figure 7 confirms that the fast mode is recommended when one needs only high update rate and doesn't care about accuracy. Moreover, it can be noted that the accuracy of the outdoor mode doesn't go over 100 millimeters up to 9 meters, but after that it exponentially grows producing readings which are far from the real value.

The last evaluation has been made observing the precision of the sensor and comparing the standard deviation of 250 consecutive readings.

Figure 8 points out a little standard deviation (therefore a good repeatability of the sensor).

Considering both figures (Figure 7 and Figure 8) we can state that the sensor is doing an unacceptable systematic error that moves the average value of the readings far from the reference value, and a less

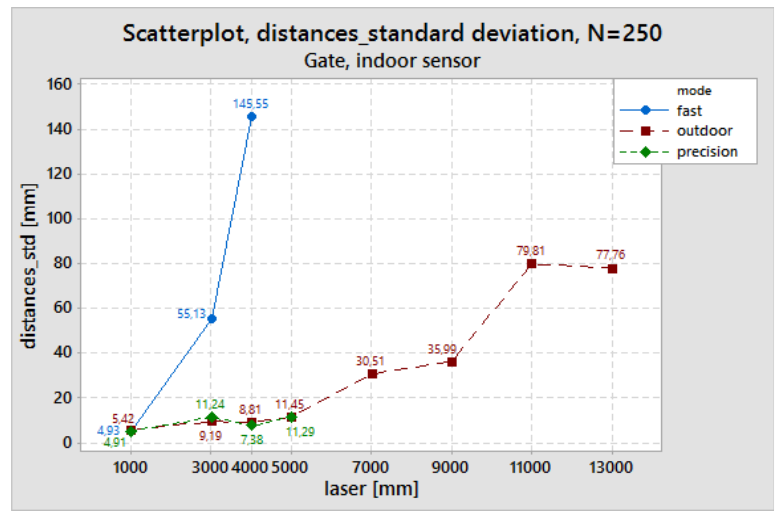

Figure 8: the standard deviation of the readings is a good indication of the sensor's precision

burdensome random error that makes acceptable the repeatability of the sensor.

This means that if the sensors were correctly calibrated shifting the average value of the readings near the real value, we would obtain a precise and 
accurate sensor that, while maintaining its best performance in indoor environment, it would be able to give good measurements also in an outdoor environment thanks to the introduction of a specific mode.

The same graphs have been created using the data of the other targets. Even if the values of the readings are obviously different, the data analysis leads to the same conclusion and confirms it. Hence, showing further results is not very significant.

\section{OUTDOOR VS INDOOR}

The introduction of the outdoor mode has increased the sensor's range in outdoor environment, but the metrological characteristics are not sufficient for Terabee. This means that there is strong need for a specific outdoor version of TeraRangerOne which fills the gaps of the indoor version.

The second part of the test related to a comparison between the outdoor sensor 1508351 and the indoor one 1508661 to understand if the new sensor (now existing only as a prototype) can further improve the outdoor measurements.

Data have been collected for each mode of the sensors, but in order to evaluate the progress of the new sensor compared to the indoor version working in outdoor mode, only graphs of this mode will be shown, and particularly the graphs relating to the

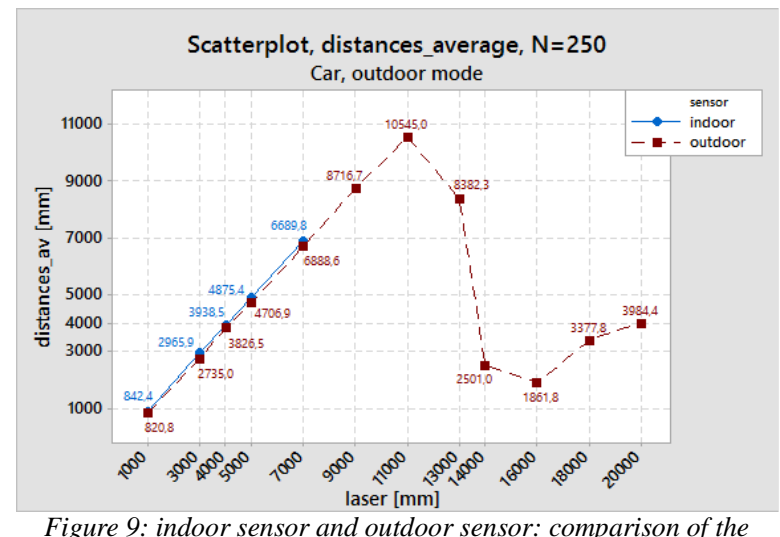

Figure 9: indoor sensor and outdoor sensor: comparison of the average readings

target "car".

Figure 9 already proves one advantage of the outdoor sensor over the indoor version. The first has in fact a range which is more than twice the indoor version's range.

The totally incorrect values over $11 \mathrm{~m}$ are not due to poor quality of the sensor, but only to its calibration. The positive thing is that the outdoor version of TeraRangerOne is able to detect the back signal even if it is 20 meters away, and this is surprising. The next step will be the sensor's calibration to combine the back signals with right readings.
If until now the improvements obtained with this sensor are not evident, it is useful to compare the

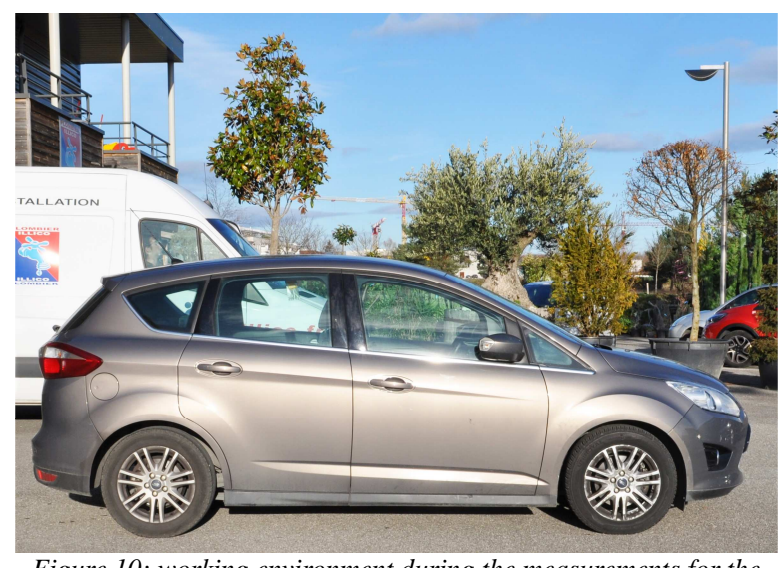

Figure 10: working environment during the measurements for the comparison of the two versions

environmental conditions during the car's measurements and the gate's measurements.

Observing Figure 10, it's easy to note that there was a strong sunlight's influence during the measurements, striking perpendicularly the target and reflecting back to the sensor. Despite the presence of optical filters, sunlight surely increases the input noise of the detector, considerably raising the detectable signal threshold.

In this extreme conditions the new sensor is able to distinguish the reflection of its impulsive LEDs signal even 20 meters away, this means that it could be able to do it in all conditions.

Someone might wonder why the graphs of the IV section show that outdoor mode of indoor sensors can go up to 13 meters while in the previous graph it is not able to detect signals beyond 7 meters. One first explanation can be guessed comparing Figure 10 with

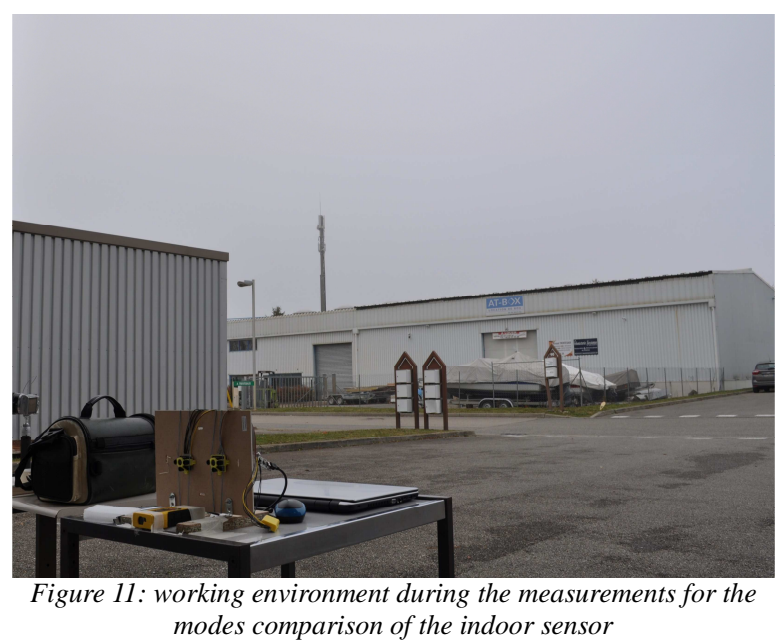

Figure 11. It can be noted that during the acquisition of the data used for the plots of section IV, the clouds 
had filtered sunrays thus reducing the receiver's input noise, and making easier to achieve a sufficient signal to noise ratio.

Moreover, also the differences between the two targets should be considered: the white gate can be taken as excellent target for its homogeneity and its high rate of infrared reflection; while the dark grey car is harder to detect because it absorbs a big part of the incident infrared light.

The environmental differences between the two tests don't restrict their meaningfulness, on the contrary it's important to test the sensors in various environmental conditions in order to perceive their limits. For example, it's useful to notice that the outdoor mode of the indoor sensor considerably decreases its range in the conditions of Figure 10. The fundamental thing is that if a comparison of two modes or of two sensors is going to be performed, then the data compared in the graphs must come from similar environmental conditions to avoid the risk of wrong conclusions.

Proceeding with data analysis, it's useful to observe Figure 12.

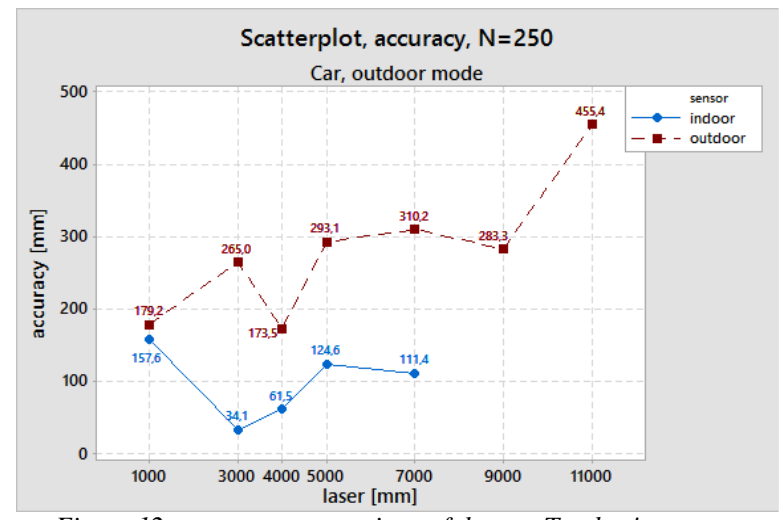

Figure 12: accuracy comparison of the two Terabee's sensors working in outdoor mode

Figure 12 clearly shows that the outdoor sensor (with the actual calibration) is still far from meeting the features which Terabee wants to obtain. In fact, at each tested distance, the indoor sensor has a better accuracy than the outdoor one, and neither of the indoor sensors' accuracy (in outdoor environment) is satisfactory for the company.

Looking at the accuracy values of the precision mode in indoor environment (published by the CERN [22]) it's easy to understand why most of the values of Figure 12 cannot be accepted. This is the confirmation of the hypothesis that outdoor sensors need a specific calibration, different from the indoor one. It's important to specify that the accuracy values have been plotted up to 11 meters only because over this threshold accuracy is bigger than 1000 millimeters and the linear graph would lose its legibility.

Same of the considerations for Figure 8 can be done for Figure 13: repeatability of new sensor is satisfying, above all in the seven initial meters where standard deviation is less than 2.1 centimeters.

This means that with an appropriate sensor calibration which moves the average readings around the real value, outdoor sensors can become the ideal sensors for any application that requires fast, precise and accurate distance measurements in outdoor environment.

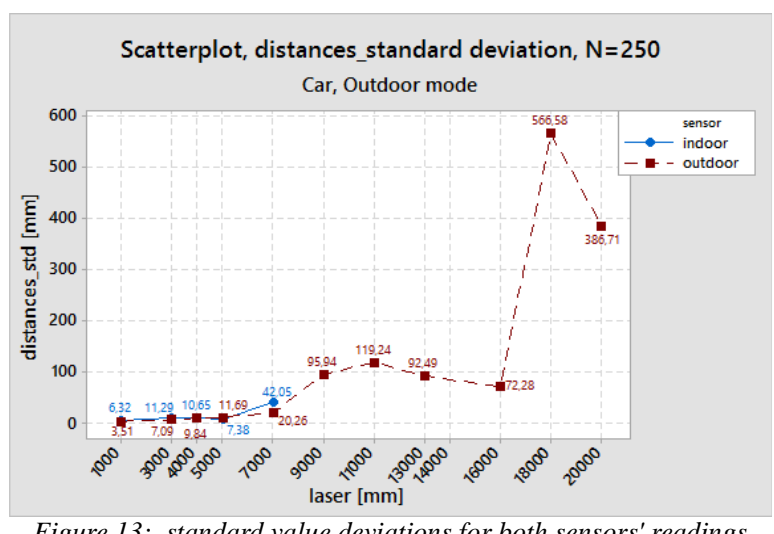

\section{CONCLUSION}

Graphs shown in this paper give some answers to the initial questions.

Certainly, a specific sensor for outdoor environment is necessary: the outdoor mode of the indoor sensor can be improved in its accuracy, but nothing can be done to increase the range of the sensor. The sensor's inability to perform measurements up to 14 meters under direct sunlight cannot be accepted. This doesn't mean that outdoor mode loses its usefulness, because the progress obtained thanks to this mode are remarkable relating to the precision and fast modes.

Anyway, for a continuous and regular use in outdoor environment, features of the indoor sensor might not be sufficient, also in outdoor mode.

Nowadays, not even the outdoor sensor has metrological characteristics which can be considered acceptable by Terabee. The potential is huge, because its range is longer than expected before the test, but the sensor's readings are too far from the real value and in order to remedy this it will be necessary to develop a new specific calibration firmware for this version. This was already planned by the company, because the new sensor uses a totally revolutionized hardware, completely different from the one of the indoor sensor.

At the moment, the TeraRangerOne is available only in its indoor version, including an outdoor mode which makes it exploitable also in outdoor environment; but in a very near future, also the outdoor sensor will be made available, and after a good calibration and a small revision, it will be effectively used in all outdoor environmental 
application, from collision avoidance systems for UAV to photogrammetry for 3-D models of buildings.

\section{REFERENCES}

[1] P. Dockrill, "Facebook is preparing its Internet-beaming drone for maiden launch," ScienceAlert. [Online]. Accessed on Jan. 17, 2016. Available: http://www.sciencealert.com/facebook-is-preparing-itsinternet-beaming-drone-for-maiden-launch

[2] G. Kimchi et al., "Unmanned aerial vehicle delivery system," U.S. Patent Application Pubblication, 2015/0120094 A1, Apr. 30, 2015.

[3] Abdullah Tariq and S M Osama, A. Gillani, "Development of a low cost and light weight UAV for photogrammetry and precision land mapping using aerial imagery," National University of Sciences and Technology (NUST), Islamabad, Pakistan, in International Conference on Frontiers of Information Technology, 2016, pp. 360-364.

[4] Maurizio Rossi and Davide Brunelli, "Autonomous Gas Detection and Mapping with Unmanned Aerial Vehicles," IEEE Transactions on Instrumentation and Measurement, vol. 65, no. 11, pp. 765-775, Apr. 2016.

[5] Paolo Tripicchio, Massimo Satler, Gustavo Stefanini, Giacomo Dabisias, Emanuele Ruffaldi and Carlo A. Avizzano, "Towards Smart Farming and Sustainable Agriculture with Drones," Scuola Superiore Sant'Anna, Pisa, Italy, in International Conference on Intelligent Environments, 2015, pp. 140-143.

[6] W. Staff, "Fighting forest fires before they get big-With drones," Wired. [Online]. Accessed on Dec. 19, 2015. Available: https://www.wired.com/2015/06/fighting-forestfires-get-big-drones/

[7] M. T. McCaul and Chairman Peter T. King, "Using Unmanned Aerial Systems Within the Homeland: Security Game Changer?," Homeland Security Committee, Washington, DC, USA, Tech. Rep. 112-107, Jul. 19, 2012.

[8] B. J. O'Brien, D. G. Baran and B. B. Luu, "Ad Hoc Networking for Unmanned Ground Vehicles: Design and Evaluation at Command, Control, Communications, Computers, Intelligence, Surveillance and Reconnaissance On-The-Move," Army Research Laboratory, Adelphi, MD, USA, ARL-TR-3991, Nov. 2006.

[9] M'arton G'otzy, D'aniel Het'enyi and L'aszl'o Bl'azovics, "Aerial surveillance system with cognitive swarm drones," Budapest University of Technology and Economics, 1117, Budapest Magyar Tud'osok k"or'utja 2 QB-207, Hungary, in Proceeding of the 28th International Conference, Cybernetics \& Informatics $(K \& I)$, Levoĉa, Slovakia, Feb. 2-5, 2016.

[10] Zainab Zaheer, Atiya Usmani, Ekram Khan and Mohammed. A. Qadeer, "Aerial Surveillance System using UAV," Department of Computer Engineering, Department of Electronics Engineering, Zakir Husain College of Engineering and Technology, Aligarh Muslim University, India, in Thirteenth International Conference on Wireless and Optical Communications Networks (WOCN), Hyderabad Campus Telangana State, India, Jul. 21-23, 2016.

[11] Mirmojtaba Gharibi, Raouf Boutaba and Steven L. Waslander, "Internet of Drones," IEEE Access, vol.4, pp. 1148-1162, Mar. 2, 2016.
[12] R. K. Sharma and D. Ghose, "Collision avoidance between UAV clusters using swarm intelligence techniques," Int. J. Syst. Sci., vol. 40, no. 5, pp. 521-538, May 7, 2009.

[13] Johann Borenstein and Yorem Koren, "Real-Time Obstacle Avoidance for Fast Mobile Robots," IEEE Transactions on Systems, Man, and Cybernetics, vol. 19, no.5, pp. 11791187, sep./oct. 1989.

[14] Jason Israelsen, Matt Beall, Daman Bareiss, Daniel Stuart, Eric Keeney and Jur van den Berg, "Automatic Collision Avoidance for Manually Tele-Operated Unmanned Aerial Vehicles," in IEEE International Conference on Robotics \& Automation (ICRA), Hong Kong Convention and Exhibition Center, Hong Kong, China, May 31 - June 7, 2014, pp. 6638-6643.

[15] Othmar Frey, Christophe Magnard, Maurice Rüegg and Erich Meier, "Focusing of Airborne Synthetic Aperture Radar Data from Highly Nonlinear Flight Tracks," IEEE Transactions on Geoscience and Remote Sensing, vol. 47, no.6, pp. 1844-1858, Jun. 2009.

[16] D. Mazierli, "Metrological characterization of new infrared sensors for robot navigation," [Italian]. Electronic and Telecommunication Engineering Bachelor Degree, Department of Information Engineering, University of Florence, Florence, Italy, Dec. 2015.

[17] G. Benet, F. Blanes, J. Simó and P. Pérez, "Using infrared sensors for distance measurement in mobile robots," Robotics and Autonomous Systems, vol. 40, no. 4, pp. 255266, 2002.

[18] Kurt Konolige, Joseph Augenbraun, Nick Donaldson, Charles Fiebig and Pankaj Shah, "A Low-Cost Laser Distance Sensor," in IEEE International Conference on Robotics and Automation, Pasadena, CA, USA, May 1923, 2008, pp. 3002-3008.

[19] T. Mohammad, "Using Ultrasonic and Infrared Sensors for Distance Measurement," International Journal of Mechanical, Aerospace, Industrial, Mechatronic and Manufacturing Engineering, vol.3, no.3, pp. 267-272, 2009.

[20] Kimberly McGuire, Guido de Croon, Christophe De Wagter, Karl Tuyls and Hilbert Kappen, "Efficient Optical Flow and Stereo Vision for Velocity Estimation and Obstacle Avoidance on an Autonomous Pocket Drone," IEEE Robotics and Automation Letters, vol.2, no.2, pp. 1070-1076, Jan. 25, 2017.

[21] A. Zanobini, "A drone anti-collision system: maintaining a fixed distance from a target during the flight," Department of Information Engineering, University of Florence, Florence, Italy, in Proceedings of the 18th Mediterranean Electrotechnical Conference, MELECON 2016, Limassol, Cyprus, Apr. 18-20, 2016.

[22] M. Ruffo, J. Kovermann, M. Di Castro, L. Molinari, R. Losito, L. Rodrigues and A. Masi, "New infrared time offlight measurement sensor for robotic platform," in 20th IMEKO TC4 International Symposium and 18th International Workshop on ADC Modelling and Testing Research on Electric and Electronic Measurement for the Economic Upturn, Benevento, Italy, Sep. 15-17, 2014, pp. 13-18.

[23] M. Baharuddin, A. Zayegh and R. Begg, "Ultrasonic and Infrared Sensors Performance in a Wireless Obstacle Detection System," in First International Conference on Artificial Intelligence, Modelling \& Simulation (AIMS), Kota Kinabalu, Sabah, Malaysia, Dec. 3-5, 2013, pp. 439444. 
